\title{
Neurofilament to predict post-anoxic neurological outcome: are we ready for the prime time?
}

\author{
Fabio S. Taccone ${ }^{1^{*}}$ (D), Jerry P. Nolan ${ }^{2,3}$ and Cornelia W. E. Hoedemaekers ${ }^{4}$
}

๑) 2020 Springer-Verlag GmbH Germany, part of Springer Nature

Among resuscitated cardiac arrest (CA) patients, hypoxic-ischaemic brain injury (HIBI) remains the main cause of mortality, with only a few potentially effective therapeutic interventions currently available [1]. As such, prognostication of neurological outcome after HIBI is of great importance because it might help physicians to either intensify care in those patients with expected neurological recovery or avoid futile interventions in those with severe and likely irreversible brain damage. A recent systematic review of prognostication studies has highlighted the complexity and difficulty in the interpretation of available data [2]. Deaths from HIBI are often mixed with deaths from other causes (e.g. cardiogenic shock or multiple organ failure). Long-term assessment can miss early neurological recovery followed by death from secondary complications (e.g. hospital-acquired infections) [3] and early withdrawal of life-sustaining therapies could result in a "self-fulfilling prophecy" and an overestimation of the predictive value of these tools for poor neurological outcome. The perfect prognostic parameter should be able to accurately separate patients with favorable and unfavorable outcomes and, if possible, achieve this in the early phase (i.e. within $48 \mathrm{~h}$ ) after the initial injury. Moreover, it should be able to quantify the extent of brain damage in a continuous and quantitative scale, rather than provide a dichotomous result (i.e. absent or present N20 cortical response to somatosensory evoked potential).

*Correspondence: ftaccone@ulb.ac.be

${ }^{1}$ Department of Intensive Care, Erasme Hospital, Université Libre de Bruxelles (ULB), Route de Lennik 808, Brussels, Belgium

Full author information is available at the end of the article
Blood biomarkers of brain injury represent a growing area of interest in this field, as they can be released from brain tissue into the bloodstream within hours following $\mathrm{CA}$ and can be assessed in the laboratory, although not easily available in clinical practice [4]. Among those, neuron-specific enolase (NSE) has been widely studied and was included in the ERC-ESICM prognostic algorithm to predict poor neurological outcome after CA [5]. Despite several studies showing that high plasma concentrations of NSE correlate significantly with long-term poor outcome $[6,7]$, no consistent threshold values ensuring the absence of false-positive prediction could be identified. This is probably because NSE values are influenced by the laboratory assay that has been used, the timing of sampling, the use of targeted temperature management (TTM) or by extra-cerebral sources, such as neuroendocrine or red blood cells. Other biomarkers, such as S-100B (i.e. glial and Schwann cells) and glial-fibrillary acidic protein (GFAP, i.e. astrocytes) have also been investigated for assessing neurological prognosis in CA patients but have no advantage over NSE [7, 8].

Neurofilaments, in particular the light chain ( $\mathrm{NfL}-$ found in axons and dendrites), are specific to the central nervous system and could be a precise surrogate marker for neuronal injury after HIBI and can also increase after different inflammatory, traumatic or degenerative cerebral diseases [9]. In this issue of Intensive Care Medicine, Wihersaari et al. showed that median NfL concentrations were significantly higher in patients with poor outcome than others after arrest [10]. The accuracy of NfL to predict neurological outcome had an area under the receiver operating characteristic curve (AUROC) of 0.98 at 24, 48 and $72 \mathrm{~h}$, which was significantly higher than NSE and S-100B. Moreover, for a sensitivity to predict poor

\section{Springer}


outcome around 99\%, specificity was impressively high (i.e. 80\%), which suggested that low NfL values could also reliably identify patients with expected favorable neurological outcome. NfL improved the performance of the predictive model including age, bystander cardiopulmonary resuscitation and the time to return of spontaneous circulation. These very promising results are consistent with two previous publications. In one study, serum NfL values were significantly higher in patients with unfavorable than in those with favorable neurological outcome and NfL values on day 7 were the most predictive of poor recovery (sensitivity of $94 \%$ and specificity of $100 \%$-Table 1) [11]. In a second large post hoc study, median serum NfL values at $24 \mathrm{~h}$ were also significantly higher in the patients with poor neurological outcome compared with the others; AUROC of NfL to predict neurological outcome was 0.94 at different time-points. NfL values also had higher sensitivity for poor neurological outcome than electroencephalography, somatosensory-evoked potentials, cerebral computed tomography and brainstem reflexes [12], as well as than NSE and/or S-100B.

What are the current limitations to the routine use of $\mathrm{NfL}$ in clinical practice after CA? First, the assay for NfL measurement is not standardized yet and, although commercially available, is not used in most of laboratories worldwide and the costs of the assay are not insignificant (30-100 €/test). Second, the threshold value maximizing the accuracy to predict neurological outcome is inconsistent across studies. Indeed, NfL values change over time and might also be influenced by the use of TTM [11]; moreover, at the same time-point, NfL thresholds were very different among studies. Thus, it remains unknown whether one single NfL measurement or repeated measurements (and trends?) over time might further improve the sensitivity of this biomarker to predict neurological outcome. Third, the extremely low NfL concentrations (i.e., $\mathrm{pg} / \mathrm{mL}$ ) may contribute to an intrinsic imprecision of the measurement assays. Fourth, only patients with outof-hospital CA in very selected patients' cohorts were included and prognostic thresholds of NfL might differ significantly for in-hospital CA or in large heterogenous populations. Other concomitant interventions, such as haemodynamic targets, may also impact on NfL concentrations. In the present study, NfL values were lower in patients treated at higher $(80-100 \mathrm{mmHg})$ than at lower mean arterial blood pressure targets $(65-75 \mathrm{mmHg})$. Fifth, whether NfL provides more prognostic information than proposed algorithms remains to be demonstrated. Although NfL concentrations would increase the prognostic accuracy of a model based on clinical variables and have higher sensitivity than other single prognostic tools, further studies should evaluate the value of adding NfL to

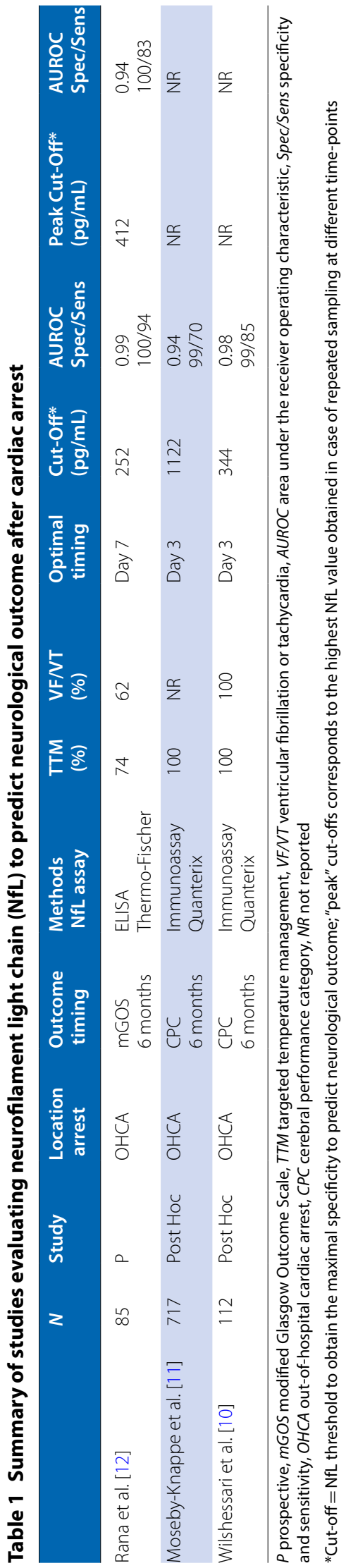


existing and validated predictive models, which include a multi-modal approach with clinical examination, electrophysiology and brain imaging [5]. Finally, more studies on the NfL thresholds to predict favorable neurological outcome would be of great interest, in particular for patients with multiple organ failure, where the implementation of additional organ support techniques, such as renal replacement therapy or extra-corporeal membrane oxygenation, could be restricted to those patients with the higher probability of neurological recovery.

Following CA, the uncertainty about patient outcomes requires an adequate prognostication approach that, given the complexity of brain function, should be based on a multi-modal algorithm. The role of available biomarkers appears to be relatively limited, but new findings on new proteins suggest their utility for early prognostication. We still ignore the pathobiological mechanisms that make NfL, which is expressed in large myelinated axons, more accurate to predict outcome than NSE, which is present in neurons, and S-100B, which is an astrocyte protein, in HIBI patients. Future prospective research needs to confirm whether NfL is the perfect biomarker for assessing neurological injury and providing reliable prognostication in this setting.

\section{Author details}

${ }^{1}$ Department of Intensive Care, Erasme Hospital, Université Libre de Bruxelles (ULB), Route de Lennik 808, Brussels, Belgium. ${ }^{2}$ Warwick Clinical Trials Unit, University of Warwick, Coventry, UK. ${ }^{3}$ Department of Anaesthesia and Intensive Care Medicine, Royal United Hospital, Bath, UK. ${ }^{4}$ Department of Intensive Care, Radboud University Nijmegen Hospital, Nijmegen, The Netherlands.

\section{Compliance with ethical standards}

\section{Conflicts of interest}

JPN is Editor in Chief of Resuscitation. FST and CWEH have no conflict of interests to declare for this work.

\section{Publisher's Note}

Springer Nature remains neutral with regard to jurisdictional claims in published maps and institutional affiliations.
Received: 15 September 2020 Accepted: 21 October 2020

Published online: 9 November 2020

\section{References}

1. Kirkegaard H, Taccone FS, Skrifvars M, Søreide E (2019) Postresuscitation care after out-of-hospital cardiac arrest: clinical update and focus on targeted temperature management. Anesthesiology 131(1):186-208

2. Sandroni C, D'Arrigo S, Cacciola S et al (2020) Prediction of poor neurological outcome in comatose survivors of cardiac arrest: a systematic review. Intensiv Care Med 46(10):1803-1851

3. Taccone FS, Horn J, Storm C et al (2019) Death after awakening from post-anoxic coma: the "Best CPC" project. Crit Care 23(1):107

4. Scolletta S, Donadello K, Santonocito C, Franchi F, Taccone FS (2012) Biomarkers as predictors of outcome after cardiac arrest. Expert Rev Clin Pharmacol 5(6):687-699

5. Sandroni C, Cariou A, Cavallaro F et al (2014) Prognostication in comatose survivors of cardiac arrest: an advisory statement from the European Resuscitation Council and the European Society of Intensive Care Medicine. Intensiv Care Med 40(12):1816-1831

6. Wiberg S, Hassager C, Stammet P et al (2017) Single versus serial measurements of neuron-specific enolase and prediction of poor neurological outcome in persistently unconscious patients after out-of-hospital cardiac arrest-a TTM-Trial Substudy. PLoS ONE 12(1):e0168894

7. Duez CHV, Grejs AM, Jeppesen AN et al (2018) Neuron-specific enolase and $\mathrm{S}-100 \mathrm{~b}$ in prolonged targeted temperature management after cardiac arrest: a randomised study. Resuscitation 122:79-86

8. Helwig K, Seeger F, Hölschermann H et al (2017) Elevated serum glial fibrillary acidic protein (GFAP) is associated with poor functional outcome after cardiopulmonary resuscitation. Neurocrit Care 27(1):68-74

9. Gaetani L, Blennow K, Calabresi P et al (2019) Neurofilament light chain as a biomarker in neurological disorders. J Neurol Neurosurg Psychiatry 90(8):870-881

10. Wihersaari L, Ashton NJ, Reinikainen M, et al. (2020) Neurofilament light chain as an outcome predictor after cardiac arrest: a post hoc analysis of the COMACARE trial. Intensive Care Med. https://doi.org/10.1007/s0013 4-020-06218-9

11. Rana OR, Schröder JW, Baukloh JK et al (2013) Neurofilament light chain as an early and sensitive predictor of long-term neurological outcome in patients after cardiac arrest. Int J Cardiol 168(2):1322-1327

12. Moseby-Knappe M, Mattsson N, Nielsen N et al (2019) Serum neurofilament light chain for prognosis of outcome after cardiac arrest. JAMA Neurol 76(1):64-71 